
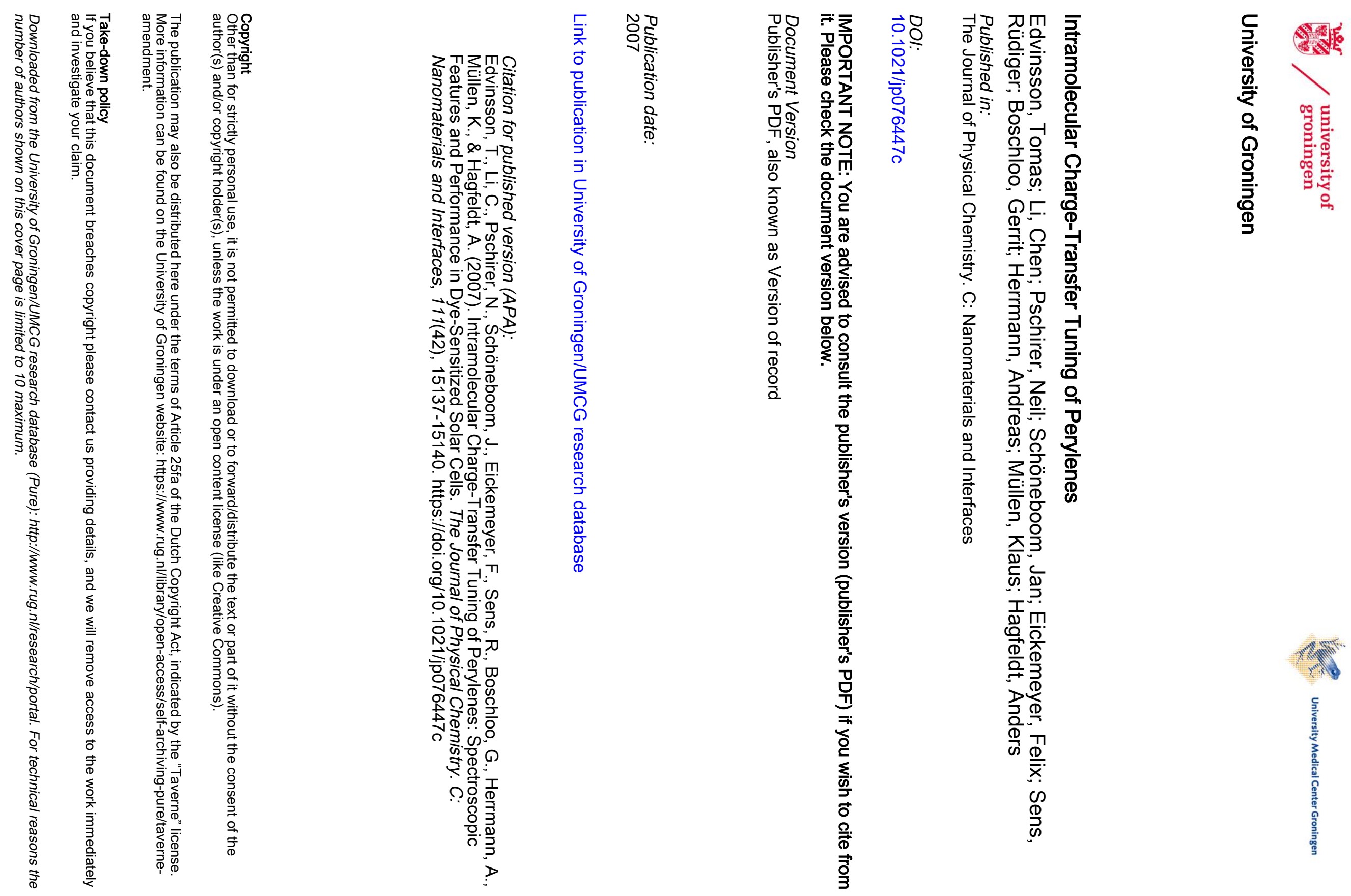


\title{
Intramolecular Charge-Transfer Tuning of Perylenes: Spectroscopic Features and Performance in Dye-Sensitized Solar Cells
}

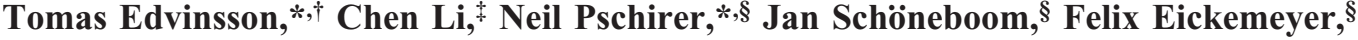 \\ Ruidiger Sens, ${ }^{\S}$ Gerrit Boschloo, ${ }^{\dagger}$ Andreas Herrmann, ${ }^{\star}$ Klaus Miillen, ${ }^{\star}$ and Anders Hagfeldt ${ }^{\dagger}$ \\ Center of Molecular Devices, Division of Physical Chemistry, Department of Chemistry, Royal Institute of \\ Technology (KTH), Teknikringen 30, 10044 Stockholm, Sweden, Max-Planck-Institute for Polymer Research, \\ Ackermannweg 10, 55128, Mainz, Germany, and BASF AG, GVP/C - A030, D-67056, Ludwigshafen, Germany
}

Received: August 10, 2007; In Final Form: September 12, 2007

\begin{abstract}
Five novel perylene molecules with different intramolecular charge-transfer (ICT) characters have been synthesized. The relation between the ICT character for different donating groups and the results for their electro- and photochemical properties as well as their performance in nanostructured dye-sensitized solar cells (nDSC) are reported. With the stronger donors, we obtain a shift of the lowest unoccupied molecular orbital (LUMO) to more negative potential versus normal hydrogen electrode (NHE) as well as an increase the charge separation in the dye upon excitation. Ab initio calculations were used to analyze the effects on orbital energies and electron distribution with the different donors. Incorporating the dyes in nDSCs, we see a drastical improvement in the performance for the more polar dyes. In particular, we find a much improved photovoltage because of higher LUMO levels, allowing conduction band tuning in the $\mathrm{TiO}_{2}$ as well as a contribution from the permanent dipoles in the dyes. The photocurrent improves remarkably with increasing ICT character of the dyes. The external quantum efficiency reached over $70 \%$, and the overall solar-toelectrical energy conversion efficiency was improved to almost $4 \%$ for the dye with highest ICT character, which can be compared with devices with the standard $\mathrm{N} 719$ dye $\left(\mathrm{Ru}(\mathrm{dcbpy})_{2}(\mathrm{NCS})_{2}\right)$ showing $6 \%$ under similar conditions. The performance is a significant improvement compared to previous reports for perylenes and lifts the performance from modest to promising. Initial stability tests show that the dye with the highest performance was spectrally stable after more than $2000 \mathrm{~h}$ of irradiation in a solar-cell device.
\end{abstract}

Nanostructured dye-sensitized solar cells (nDSC) have attracted a great deal of interest because of their efficient conversion of solar energy to electricity at a low cost. ${ }^{1,2}$ Dyesensitized solar cells based on bi- and polypyridyl ruthenium complexes have achieved solar-to-electrical energy conversion efficiencies of $10-11 \%$ under AM 1.5 irradiation. ${ }^{3-5}$ In recent years, there has been much effort in replacing the ruthenium complexes with fully organic photosensitizers for environmental reasons, lower cost, and the possibility to obtain very high extinction coefficients, which could also allow application in thinner solar cells as demanded in, for example, solid-state nDSCs. Many different dye classes have been investigated, such as coumarines,${ }^{6-8}$ xanthenes, ${ }^{9}$ porphyrins,${ }^{10,11}$ polyenes, ${ }^{12-15}$ and indolines, ${ }^{16-18}$ yielding respectable conversion efficiencies between 5 and $9 \%$ with the traditional iodide/triiodide redox system, while less has been reported on their photostability.

Here, we present the tuning of the intramolecular charge transfer (ICT) character of a very stable dye class and report the corresponding change in spectral and electrochemical properties. Rylenes are well-known for their high extinction

* Corresponding authors. E-mail: Tomased@kth.se; neil.pschirer@ basf.com.

† Royal Institute of Technology (KTH).

Max-Planck-Institute for Polymer Research.

$\S$ BASF AG. coefficients and their exceptional photophysical and photochemical stability both as aggregates as well as at the single molecular level. ${ }^{19}$ Five dyes are reported here (see Figure 1), one of which being relatively nonpolar, the other four with varying degrees of CT character (the synthesis procedures are found in the Supporting Information).

Energy-level matching is crucial in designing new sensitizers. The formal oxidation potentials of the dyes measured by squarewave voltammetry (SWV) are listed in Table 1. Cyclic voltammetry was also performed (not reported) to verify the reversibility of the processes and was within $0.02 \mathrm{~V}$ of the SWV measurements. The relative symmetric perylene-imide ID17 has a significantly higher oxidation potential of $1.60 \mathrm{~V}$ versus NHE as compared to the amino-substituted derivatives, which fall into the range between 1.15 (ID35) and 1.01 (ID22) $\mathrm{V}$ versus NHE. From an analysis of the density functional theory (DFT) orbitals, it is apparent that the amino substituent acts as an effective $\pi$-donor, contributing to the highest occupied molecular orbital (HOMO) and thereby raising the orbital energy while lowering the oxidation potential. In all of the dyes, however, the oxidation potential is more positive than the redox potential of $\mathrm{I}^{-} / \mathrm{I}_{3}{ }^{-}$(ca $0.40 \mathrm{~V}$ vs NHE), thus providing enough driving force for an efficient regeneration to the neutral sensitizer state when functionalized in a nDSC device with $\mathrm{I}^{-} / \mathrm{I}_{3}{ }^{-}$. 

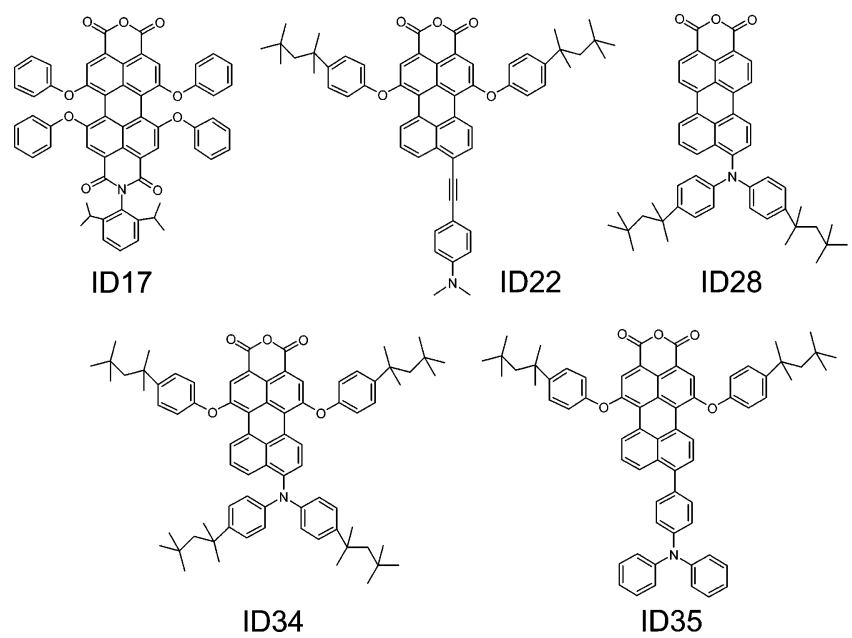

Figure 1. Molecular structures of the new perylene dyes.

TABLE 1: Electrochemical and Spectroscopic Data for the Perylene Dyes in Dichloromethane Solution and Adsorbed on $\mathrm{TiO}_{2}($ Denoted with *)

\begin{tabular}{|c|c|c|c|c|c|}
\hline dye & $\begin{array}{c}E_{\text {ox }} / \mathrm{V} \\
\text { vs } \\
\text { NHE }\end{array}$ & $\begin{array}{c}\epsilon_{\max }\left(\lambda_{\max }\right) / \\
\mathrm{M}^{-1} \mathrm{~cm}^{-1}(\mathrm{~nm})\end{array}$ & $\begin{array}{c}\lambda_{\max } * / \\
\mathrm{nm}\end{array}$ & $\begin{array}{c}E_{0-0} * / \mathrm{eV} \\
\exp ^{a}\left(\operatorname{calc}^{b}\right)\end{array}$ & $\begin{array}{l}\text { LUMO }^{*} \\
\text { open exp } \\
\left(\text { calc }^{d}\right)\end{array}$ \\
\hline ID17 & 1.60 & & 527 & 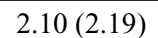 & J) \\
\hline & & & & & \\
\hline ר? & 1.1 & & & & $1(-$ \\
\hline & & & & & \\
\hline ID35 & 1.15 & $35000(540)$ & 430 & $2.41(2.41)$ & $-1.3(-1$. \\
\hline
\end{tabular}

${ }^{a}$ The experimental $E_{0-0}$ is estimated from the absorption onset of the dyes adsorbed on $\mathrm{TiO}_{2}$ at $10 \%$ of the full amplitude at the absorption maximum. ${ }^{b}$ Lowest TDDFT calculated excitation energies of disodium salts as model systems. ${ }^{c}$ Obtained as the sum of experimental $E_{\text {redox }}$ and $E_{0-0}$ for the dye on $\mathrm{TiO}_{2}$ expressed in V vs NHE. ${ }^{d}$ The theoretical values were normalized to the experimental datum of ID17.
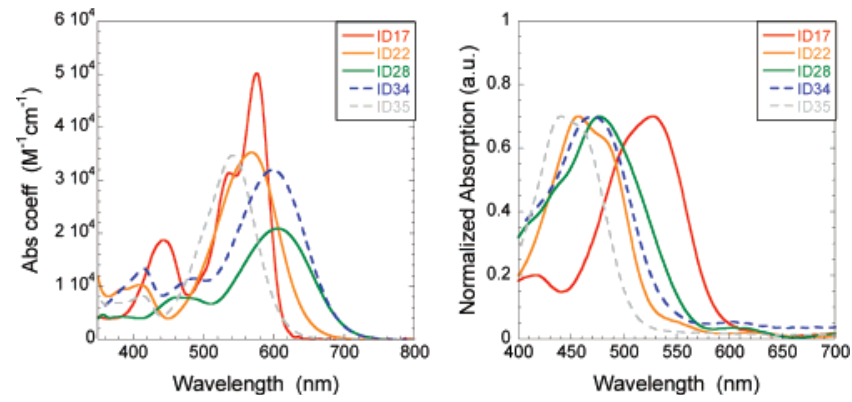

Figure 2. Absorption spectra for the perylenes in dichloromethane solution (left) and adsorbed on $\mathrm{TiO}_{2}$ (right).

The absorption spectra of the dyes in solution and adsorbed on $\mathrm{TiO}_{2}$ are shown in Figure 2, and $\lambda_{\max }$ values are summarized in Table 1. In solution, a broadening of the $S_{1}$ transition as well as a decrease in the absorption coefficient is observed when going from the relatively symmetrical ID17 to the more polarized dyes. Going from ID17 $\rightarrow$ ID22 $\rightarrow$ ID28, a monotonic decrease of the absorption coefficient and a red-shift of the absorption on-set are observed because of increasing donor strength (see Figure 2). Trying to retain both a higher absorption coefficient and a red-shifted absorption, we added bay-substituents, for example, ID34 retains the spectral signature of ID28 while improving the absorption coefficient. Changing the donor group on ID34 to a triphenylamine, ID35 is obtained with a weaker donating ability because of the spatial separation of the amine from the perylene chromophore. For ID35, we thus see a higher absorption coefficient and a blue shift compared to ID34.
All dyes show a blue-shifted absorption when adsorbed onto $\mathrm{TiO}_{2}$. This effect is attributed to the ring opening of the anhydride group on perylene to form two carboxylates, providing interactions with the oxide surface. The same blue shifts are observed upon treatment of the dyes with $\mathrm{Na}_{2} \mathrm{CO}_{3}$ in THF solution, forming the disodium salts of the sensitizers. Quantum mechanical calculations in the Turbomole program, using timedependent density functional theory (TD-DFT) calculations at the B3-LYP/def-SV(P) level, show a blue-shifted absorption of the disodium salts as a result of reduced effective conjugation length. Analyses of the theoretical results also show the decrease of the effective conjugation for all dyes and a decrease of the absolute dipole moment with respect to their anhydride analogous for all dyes except for the symmetrical ID17. The blue shift can be attributed to (i) a smaller conjugation length of the ring-opened forms, effective in all dyes and (ii) a decreased polarity, effective in the donor-substituted dyes (Table 1).

Because the decrease in effective conjugation length upon ring opening is the same in all perylene units, the shift of absorption wavelength upon ring opening may be taken as a measure of the intramolecular charge-transfer character toward the region of the anhydrous group/attachment group of the different dyes. The dyes can be arranged in an effective directive charge-transfer scale by taking the difference for the absorption onset for the ring-closed form in solution and the absorption onset for the dyes adsorbed on $\mathrm{TiO}_{2}$ (experimental values). We arrive at ID17 $\rightarrow$ ID35 $\rightarrow$ ID22 $\rightarrow$ ID34/ID28 in terms of increased intramolecular charge-transfer character. The DFT calculations show a very similar electron distribution for the LUMO on the perylene core for the polarized dyes (ID35, ID22, ID34, ID28). We also note the successivly increased redistribution of electrons from the donor group in the ground state (HOMO) to the perylene core upon excitation (LUMO) in the series for the anhydride open form of the dyes, as seen in Figure 3. Assuming similar orbital distribution in the dyes when attached to $\mathrm{TiO}_{2}$, the electronic coupling of the LUMO in the different polarized dyes and the $\mathrm{TiO}_{2} 3 \mathrm{~d}$ orbitals would be very similar, whereas the charge separation upon excitation is much improved in the series, consistent with the experimental ICT series. This would then prolong the excited-state lifetime in the dye (D) and increase the yield for charge transfer to $\mathrm{TiO}_{2}$ in competition with $\mathrm{D}^{*} \rightarrow \mathrm{D}$ recombination. The driving force for injection is estimated to be very similar for the more polarized dyes because both the electron distribution in the LUMO would give similar electronic coupling and distance to $\mathrm{TiO}_{2}$ and the LUMO energy levels are at similar energy levels (see Figure 3 and Table 1). The DFT calculated dipole moment vectors show that the polarity of the amino-substituted dyes (ID22, ID28, ID34, ID35) is retained (although reduced in magnitude from $9-12$ to $5-6$ D) in the salt versions, while the polarity of ID17 is essentially inverted in its disodium salt and the polarity is increased from 3 to $9 \mathrm{D}$ (see the Supporting Information). We can conclude that an essential part of the ground-state polarity in the amino-substituted sensitizers is also retained when ringopened.

Incorporation of the dyes into $\mathrm{nDSC}$ devices resulted in high external quantum efficiencies (EQEs) for photocurrent generation, $>70 \%$ (Figure $4 \mathrm{a}$ ).

Addition of 4-tert-butylpyridine (4-TBP) to the electrolyte, known to shift the $\mathrm{CB}$ to more negative potentials, ${ }^{20}$ reduced the $E Q E$ values (Figure $4 \mathrm{~b}$ ) for all dyes as a result of a lower driving force for electron injection, while only a modest blueshift for the photocurrent onset was observed. The lower value for ID17 is explained by electron injection limitations resulting 

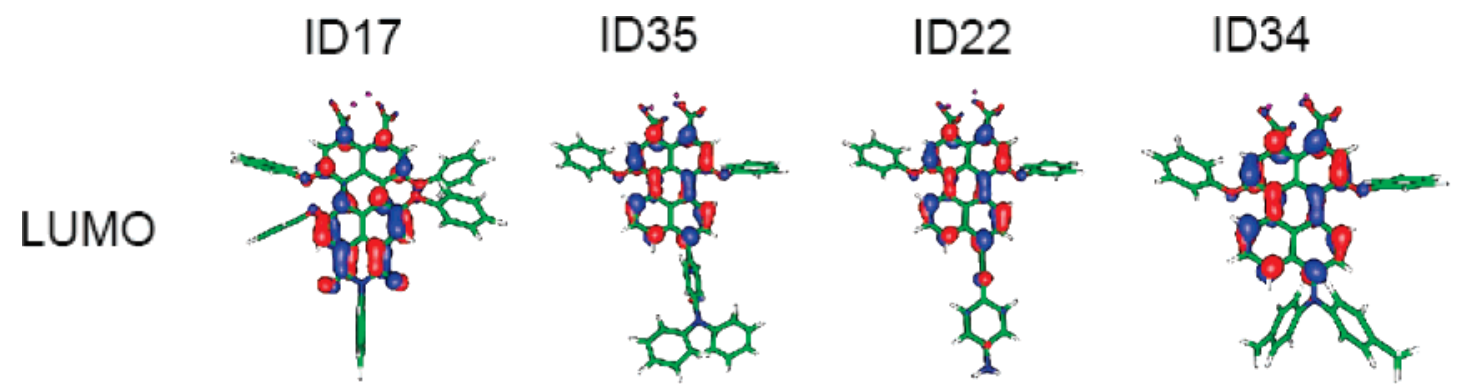

ID28
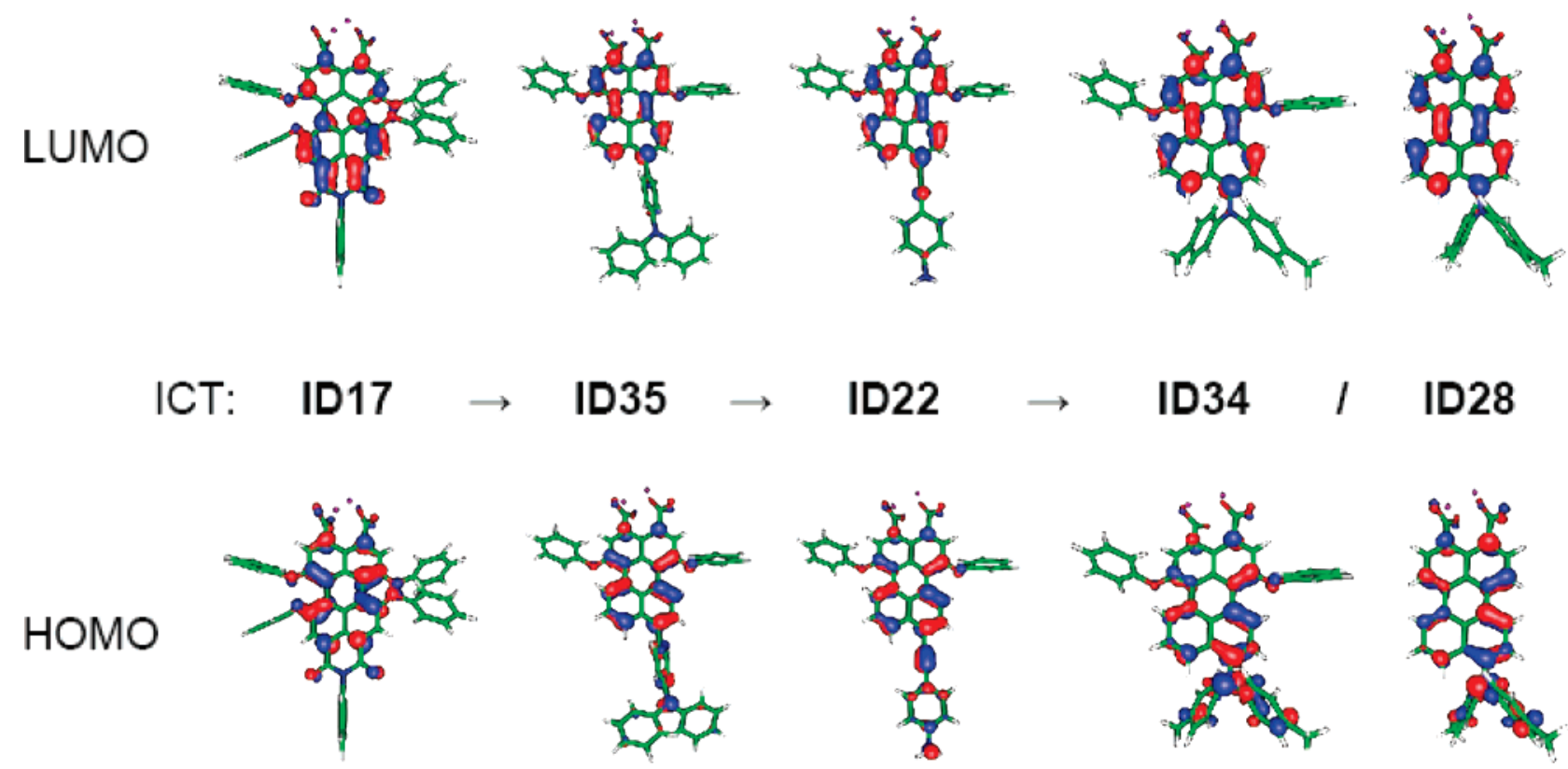

Figure 3. Optimized electronic structure and HOMO and LUMO electron density of the ring-opened (disodium salt) of the perylenes, obtained with TD-DFT calculations at the B3-LYP/def-SV(P) level. The HOMO-LUMO transition showed the strongest oscillator strength and represents the main transition at the absorption maximum. Alkyl groups are omitted with neglectable effects on the electronic structure and relative energies.
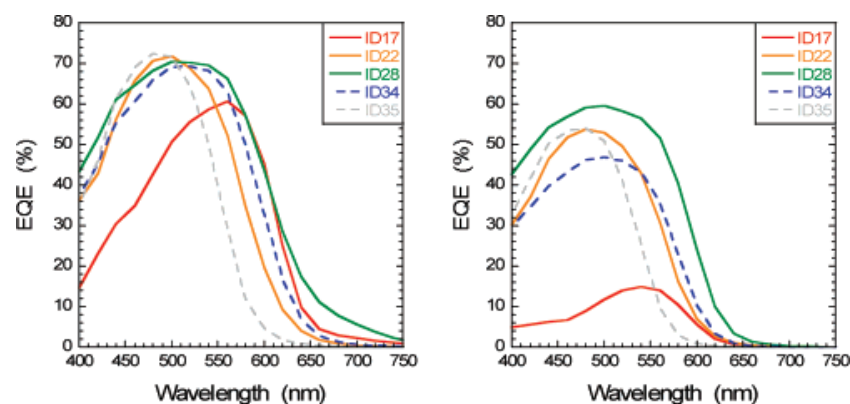

Figure 4. External quantum efficiency for dye-sensitized $\mathrm{TiO}_{2}$ using (a) an electrolyte consisting of TBAI $(0.25 \mathrm{M})$, LiI $(0.5 \mathrm{M})$, and $\mathrm{I}_{2}$ $(0.05 \mathrm{M})$ in 3-MPN and (b) an electrolyte with TBAI (0.6 M), LiI (0.1 $\mathrm{M}), \mathrm{I}_{2}(0.05 \mathrm{M})$, and $4-\mathrm{TBP}(0.5 \mathrm{M})$ in 3-MPN.

from the significantly lower lying LUMO energy compared to the other, more polarized dyes. The LUMO of ID35, for example, was measured to be $0.8 \mathrm{eV}$ higher (calculation gives $0.6 \mathrm{eV}$ ) than ID17. The additive improved, however, the photovoltage of the devices. The best efficiencies were measured when incorporating the 4TBP additive for all dyes except ID17. The measured efficiencies $\mathbf{1 . 4 \%} \rightarrow \mathbf{2 . 0 \%} \rightarrow \mathbf{2 . 4 \%} \rightarrow \mathbf{3 . 2} \%$ / $\mathbf{3 . 9 \%}$ followed the trend of the ICT values above, especially regarding $I_{\mathrm{sc}}\left(6.1 \rightarrow 7.5 \rightarrow 8.0 / 10.4 \mathrm{mAcm}^{-2}\right)$ of the polarized dyes (Table 2). Recalling the ICT analysis, it thus seems that the ICT character improves the injection efficiency and thereby improves the photocurrent. The molar amount of dye in the devices does not correlate with the observed photocurrent, where the dye content is, for example, largest for ID22 (dye coverage data are found in the Supporting Information).

Furthermore, the polarized dyes show much higher $V_{\text {oc }}$ values (598-696 mV) when compared to ID17 (444 mV) because of the possibility of using 4-TBP in the electrolyte. The calculated dipoles for the dyes in the open form are 5-6 D for the polarized dyes, and are in the same order as the strongest dipoles investigated as electrolyte additives for $\mathrm{nDSC}$ in other studies. $^{21,22}$ We can thus also expect a contribution to the higher $V_{\text {oc }}$ from the dyes themselves, giving a $\mathrm{CB}$ shift from the
TABLE 2: Device Performance from $E Q E$ and $I V$ Measurements

\begin{tabular}{ccccccc}
\hline dye & $\begin{array}{c}E Q E_{\max }^{a /} \\
\%\end{array}$ & $\begin{array}{c}E Q E_{\max }{ }^{b /} \\
\%\end{array}$ & $\begin{array}{c}V_{\mathrm{OC}^{b /}} \\
\mathrm{mV}\end{array}$ & $\begin{array}{c}I_{\mathrm{SC}^{b}} \\
\mathrm{~mA} / \mathrm{cm}^{2}\end{array}$ & $\begin{array}{c}\text { fill factor } \\
\%\end{array}$ & $\begin{array}{c}\eta^{b, c /} \\
\%\end{array}$ \\
\hline ID17 & 61 & 15 & $444^{a}$ & $7.32^{a}$ & $43^{a}$ & $1.4^{a}$ \\
ID22 & 71 & 54 & 598 & 7.50 & 54 & 2.4 \\
ID28 & 70 & 60 & 651 & 10.4 & 58 & 3.9 \\
ID34 & 69 & 47 & 696 & 7.96 & 59 & 3.2 \\
ID35 & 72 & 54 & 651 & 6.13 & 55 & 2.2
\end{tabular}

${ }^{a}$ Electrolyte: $0.25 \mathrm{M}$ TBAI, $0.5 \mathrm{M}$ LiI, $0.05 \mathrm{M} \mathrm{I}_{2}$ in 3-MPN. ${ }^{b}$ Electrolyte: $0.6 \mathrm{M}$ TBAI, 0.1 M LiI, $0.05 \mathrm{M} \mathrm{I}_{2}$, and $0.5 \mathrm{M}$ 4-TBP in acetonitrile. ${ }^{c}$ Devices were made without scattering layers, $\mathrm{TiCl}_{4}$ treatment, or antireflecting coatings and were fully masked to an exact illumination area during the measurements. The values can be compared to $\eta=5.8-6.0 \%$ obtained for $\mathrm{nDSC}$ devices with the ruthenium complex N719 made under the same conditions.

increased dipole at the $\mathrm{TiO}_{2} /$ dye interface 21,22 as well as from hindering the recombination of electrons from the $\mathrm{TiO}_{2} \mathrm{CB}$ to the oxidized dye.

Current-voltage behavior in dark revealed that ID17 showed dark currents already at low potentials, whereas the devices with ID22, ID28/ID35, and ID34 showed dark currents at successively higher potential (data given in the Supporting Information). This is in excellent agreement with the increased photovoltage in Table 2. The reduction in dark current at a certain potential can originate from (i) higher $\mathrm{CB}$ in the $\mathrm{TiO}_{2}$ and thus less electron population in the $\mathrm{CB}$ or (ii) improved electronic blocking in between $\mathrm{I}_{3}{ }^{-}$species in the electrolyte and the $\mathrm{F}: \mathrm{SnO}_{2} /$ dye and $\mathrm{TiO}_{2} /$ dye interface. To attribute this effect to the molecular origin, apart from the dipole effect, we can also note the alkyl groups at both the donor group and the bay positions in ID34, giving the least dark current. In part, repulsion of $\mathrm{I}_{3}{ }^{-}$species from the $\mathrm{TiO}_{2}$ surface can also contribute to a suppressed dark current in this case. However, the specific molecular origin of the improved dark current behavior and the relation between the $\mathrm{CB}$ shift versus the improved electron lifetime are not certified at this stage and more investigation with, for example, electron lifetime measurements are needed. 
Other factors, such as an increasing hole-mobility in di- and triarylamine groups acting as electron acceptors at the unprotected $\mathrm{F}: \mathrm{SnO}_{2}$ back contact could also affect the overall performance in the solar-cell devices. Different driving forces for regeneration via the different redox potentials would be less critical because all of the dyes have $0.6 \mathrm{~V}$ or more in driving force for regeneration.

Combining experimental studies and quantum mechanical calculations, we infer that these donors act in two ways: (i) generally raising the LUMO energy level, thus providing a more efficient injection of electrons into the conduction band (CB) of $\mathrm{TiO}_{2}$ and allowing $\mathrm{CB}$ tuning through the use of additives to give more voltage, and (ii) successively improving the intramolecular charge separation in the dye, promoting more effective electron injection into $\mathrm{TiO}_{2}$ in competition with $\mathrm{D}^{*} \rightarrow \mathrm{D}$ recombination and preventing the undesired recombination of electrons from the $\mathrm{TiO}_{2} \mathrm{CB}$ with the oxidized dye. The donors also polarize the dyes to permanent dipoles, which is beneficial for the nDSC performance, most markedly seen in the improved photovoltage. Assuming that the dye is adsorbed in monolayers, dye coverage alone cannot explain the observed dark current behavior. The molecular origin of this effect can in part be explained by a contribution of the dye dipoles to a $\mathrm{CB}$ shift and/or repulsion of $\mathrm{I}_{3}{ }^{-}$species from the $\mathrm{TiO}_{2}$ surface, resulting in less recombination.

Previous studies on perylene dyes in $\mathrm{nDSC}$ have so far resulted in overall solar-to-energy conversion efficiencies $(\eta)$ below $1 \%,{ }^{23-25}$ temporarily increased to $1.2-2.0 \%$ after UVtreatment of the solar cell. ${ }^{26-28}$ In these studies, tight energy matching with the dye LUMO and metal oxide CB limits the possible attainable photovoltage to $300-500 \mathrm{mV}$. Recently, up to $2.6 \%$ efficiency has been reported ${ }^{28}$ but limited by low external quantum efficiency $(<40 \%)$ and in part also limited by low photovoltage $(540 \mathrm{mV})$. In this work, the introduction of stronger donating groups (i.e., diphenyl amines, ID28, 34) attached to the perylene core at the point furthest away from the anchor group push up the LUMO and create relatively strong dipole moments (5-6 D in the open forms) in the perylenes. The photocurrent increased with the ICT character of the dyes, and the more polar perylenes show a strongly improved photovoltage in nDSC devices (up to $\sim 700 \mathrm{mV}$ ). The dyes reach external quantum efficiencies over $70 \%$ and up to $4 \%$ in overall solar-to-electricity conversion efficiency without any UV treatment. The devices are made without scattering layers, $\mathrm{TiCl}_{4}$ treatment, or antireflecting coatings and with less internal area than fully optimized $\mathrm{TiO}_{2}$ electrodes and can be compared to solar cells made with the ruthenium complex N719, giving up to $6 \%$ under similar conditions.

Preliminary stability tests show stable absorption spectra of ID28 in nDSC devices during irradiation for $2000 \mathrm{~h}$ at ambient temperature. The approach to start with inherently stable organic photomaterial and then alter the properties to improve the performance in photovoltaic devices can be an interesting route toward functional dye-design.

Supporting Information Available: Detailed results and technical data concerning the synthesis, characterization, DFT calculations, device fabrication, and dye load. This material is available free of charge via the Internet at http://pubs.acs.org.

\section{References and Notes}

(1) Hagfeldt, A.; Grätzel, M. Chem. Rev. 1995, 95, 49

(2) Hagfeldt, A.; Grätzel, M. Acc. Chem. Res. 2000, 33, 269-277.

(3) O’Regan, B.; Grätzel, M. Nature. 1991, 353, 737

(4) Nazeeruddin, M. K.; Kay, A.; Rodicio, I.; Humphrybaker, R.; Muller, E.; Liska, P.; Vlachopoulos, N.; Grätzel, M. J. Am. Chem. Soc. 1993, 115,6382 .

(5) Grätzel, M. J. Photochem. Photobiol. 2004, 164, 3.

(6) Hara, K.; Sayama, K.; Ohga, Y.; Shinpo, A.; Suga, S.; Arakawa, H. Chem. Commun. 2001, 6, 569.

(7) Hara, K.; Wang, Z.-S.; Sato, T.; Furube, A. J. Phys. Chem. B 2005 , $109,15476$.

(8) Hara, K.; Kurashige, M.; Dan-oh, Y.; Kasada, C.; Shinpo, A.; Suga, S.; Sayama, K.; Arakawa, H. New J. Chem. 2003, 27, 783.

(9) Hara, K.; Horiguchi, T.; Kinoshita, T.; Sayama, K.; Sugihara, H.; Arakawa, H. Chem. Lett. 2000, 29, 316.

(10) Tokuhisa, H.; Hammond, P. T. Adv. Funct. Mater. 2003, 13, 831

(11) Nazeeruddin, Md. K.; Humphry-Baker, R.; Officer, D. L.; Campbell, W. M.; Burrell, A. K.; Grätzel, M. Langmuir. 2004, 20, 6514.

(12) Kim, S.; Lee, J. K.; Kang, S. O.; Ko, J.; Yum, J. H.; Fantacci, S.; De Angelis, F.; Di Censo, D.; Nazeeruddin, M. K.; Grätzel, M. J. Am. Chem. Soc. 2006, 128, 16701.

(13) Hagberg, D. P.; Edvinsson, T.; Marinado, T.; Boschloo, G.; Hagfeldt, A.; Sun, L. C. Chem. Commun. 2006, 2245.

(14) Hara, K.; Sato, T.; Katoh, R.; Furube, A.; Yoshihara, T.; Murai, M.; Kurashige, M.; Ito, S.; Shinpo, A.; Suga, S.; Arakawa, H. Adv. Funct. Mater. 2005, 15, 246.

(15) Kitamura, T.; Ikeda, M.; Shigaki, K.; Inoue, T.; Anderson, N. A.; Ai, X.; Lian, T. Q.; Yanagida, S. Chem. Mater. 2004, 16, 1806.

(16) Horiuchi, T.; Miura, H.; Uchida, S. Chem. Commun. 2003, 3036.

(17) Horiuchi, T.; Miura, H.; Sumioka, K.; Uchida, S. J. Am. Chem. Soc. 2004, 126, 12218 .

(18) Ito, S.; Shaik, M.; Zakeeruddin, S. M.; Humphry-Baker, R.; Liska, P.; Charvet, R.; Comte, P.; Nazeeruddin, M. K.; Péchy, P.; Takata, M.; Miura, H.; Uchida, S.; Grätzel, M. Adv. Mater. 2006, 18,1202

(19) Dubois, A.; Canva, M.; Brun, A.; Chaput, F.; Boilot, J.-P. Appl. Opt. 1996, 35, 3193.

(20) Boschloo, G.; Haeggman, L.; Hagfeldt, A. J. Phys. Chem. B 2006 110,13144 .

(21) Rühle, S.; Greenshtein, M.; Chen, S.-G.; Merson, A.; Pizem, H.; Sukenik, C. S.; Cahen, D.; Zaban, A. J. Phys. Chem. B 2005, 109, 18907 (22) Krüger, J.; Bach, U.; Grätzel, M. Adv. Mater. 2000, 12, 447

(23) Ferrere, S.; Zaban, A.; Gregg, B. A. J. Phys. Chem. B 1997, 101, 4490.

(24) Wang, S.; Lia, Y.; Dua, C.; Shia, Z.; Xiaoa, S.; Zhua, D.; Gaob, E.; Cai, S. Synth. Met. 2002, 128, 299.

(25) Dentani, T.; Funabiki, K.; Jin, J.-Y.; Yoshida, T.; Minoura, H.; Matsui, M. Dyes Pigm. 2007, 72, 303.

(26) Ferrere, S.; Gregg, B. A. J. Phys. Chem. B. 2001, 105, 7602

(27) Ferrere, S.; Gregg, B. A. New J. Chem. 2002, 26, 1155.

(28) Shibano,Y.; Umeyama, T.; Matano, Y.; Imahori, H. Org. Lett. 2007, 9, 1971 